\title{
AVALIAÇÃO MÉDICA: \\ O CONSUMO NA MEDICINA E A MERCANTILIZAÇÃO DA SAÚDE
}

\author{
JÚLIO CÉSAR MONTEIRO DOS SANTOS JR ${ }^{1}$
}

${ }^{1}$ Hospital e Maternidade Frei Galvão de Guaratinguetá

SANTOS JR JCM. Avaliação Médica: O Consumo na Medicina e a Mercantilização da Saúde. Rev bras Coloproct, 2006;26(1):70-85.

RESUMO: Introdução: A Saúde vem sendo ameaçada pela colonização empresarial do médico que, iniciada dentro da Universidade, prolonga-se no ambiente de trabalho. Essa ação tem origem no ensino defasado da realidade científica e na asserção de tendências individualistas que expressam opiniões isoladas e não abalizadas, em geral induzidas pelas propagandas e investigações encomendadas para agitar o mercado no uso de aparelhos e produtos médico-farmacêuticos.

Objetivo: Usar o ponto de vista do Cirurgião Geral e do Coloproctologista para comentar a mercantilização da saúde e a maneira como a Instituição Industrial, usando a empresa médica, age e modifica a ação do médico, contribuindo para o alto custo da Medicina.

Material e método: As bases serão os exames laboratoriais e as avaliações cardiovasculares pré-operatórias de rotina, usados para operações não cardíacas em pacientes cardiopatas. O material e o conteúdo para discussão foram extraídos do livro de Ivan Illich ${ }^{1}$, do artigo de atualização de Coelho e col. ${ }^{2}$, do modelo proposto na Cleveland Clinic ${ }^{3}$ sobre avaliação pré-operatória e das normas estabelecidas pelo American College of Cardiology e pela American Heart Association ${ }^{4}$ para a orientação de avaliação médica mínima, necessária e suficiente, de pacientes cardiopatas, quando candidatos a tratamento cirúrgico de doenças em outros órgãos.

Descritores: Avaliação pré-operatória; exames laboratoriais; operações não cardíacas; mercantilização da medicina, expropriação da saúde

“Exames de laboratórios para alguns médicos e para os pacientes, de um modo geral, são bens de consumo."

\section{INTRODUÇÃO}

Tendo deixado as atividades universitárias, instalamo-nos numa cidade do interior para viver uma nova etapa de vida profissional, exercendo as especialidades de Cirurgia Geral, Coloproctologia e Endoscopia Digestiva.

No início, constituímos equipe médica formada por pessoas com propósitos afins, grupo para o qual convidamos um médico anestesiologista. Oferecemos ao colega um consultório no mesmo ambiente físico onde se instalaram três cirurgiões gerais, um oncologista cirúrgico, dois coloproctologistas, um cirurgião vascular, um gineco-obstetra, um neurocirurgião, um psiquiatra e uma neonatologista.

A proposta estava vinculada, de acordo com nossa opinião, à necessidade de complementação da avaliação pré-operatória e ao fato insólito de que, no nosso novo ambiente de trabalho, a consulta préanestésica era feita na sala operatória, momentos antes da indução anestésica.

Trabalho realizado no Hospital e Maternidade Frei Galvão de Guaratinguetá

Recebido em $03 / 03 / 2006$

Aceito para publicação em 27/03/2006 
Ali, o médico anestesiologista se apresentava ao paciente, em geral, com um questionário longo, mas desenvolvido de maneira rápida e cheio de falhas, às vezes, buscando para complementar sua avaliação o auxílio do cirurgião responsável que, respondendo sobre questões fundamentais, nem sempre era capaz de aliviar as dúvidas do médico anestesista.

Dois anos depois passamos a contar com o trabalho do anestesiologista, na avaliação préanestésica.

Atualmente, temos observado um outro tipo de impasse. Preparado pelo cirurgião para a operação, o paciente tem sido encaminhado para consulta denominada de avaliação pré-anestésica. No contato com o médico anestesiologista, o paciente responde a um questionário semelhante aos elaborados, como sugestão, pelas Sociedades Médicas Especializadas. Ao término da consulta, o paciente recebe uma guia de solicitação de exames, nem sempre condizentes com as necessidades ou compatíveis com o problema de saúde apresentado pelo doente.

A situação piora quando o paciente tem uma ou mais doenças associadas, mormente quando a associação é uma cardiopatia. Nesses casos, é óbvio, adiciona-se às avaliações anteriores a justa participação do médico cardiologista, mas antes deste, acrescenta-se também uma nova série de exames que, quando não em discordância com a situação de enfermidade em que se encontra o paciente, excede as necessidades básicas para a avaliação que se pretende.

Acreditamos que o problema é geral: cria desconforto entre os membros da equipe, eleva os custos da ação médica, favorece em certos aspectos a instituição, inviabiliza o sistema público, proporciona instabilidade emocional ao paciente, além de não the assegurar melhor atendimento.

Apostando na afirmação de que "a avaliação pré-operatória é essencial para o sucesso dos procedimentos cirúrgicos" ${ }^{2}$, voltamos ao tema, motivo de debates em outros lugare ${ }^{1-4}$ pelo interesse que desperta nos cirurgiões, em geral, e, em especial, nos coloproctologistas, principalmente pelos dois motivos citados: o primeiro, acrescenta custos que ultrapassam a medida justa e, o segundo, não traz benefícios à equipe médica ou ao paciente. ${ }^{2,3} \mathrm{~A}$ esses motivos, acrescentamos um terceiro que é o potencial aumento de prejuízo à saúde do paciente.
Os gastos anuais com exames laboratoriais para avaliação pré-operatória, nos EUA, atingem a cifra de 30 bilhões de dólares e têm sido sistematicamente questionados quanto à validade ${ }^{5}$ pelos aspectos da indiscriminação, da falta de justificativas e nenhum benefício auferido pelos pacientes, em termos de segurança e melhores resultados atingidos pelo tratamento proposto. ${ }^{2,3}$

Estima-se que $60 \%$ dos exames ditos de "rotina" podem ser dispensados, sem qualquer risco para os pacientes'; soma-se, ainda, a observação de que as alterações detectadas nesses exames poderiam ser previstas na avaliação clínica ${ }^{3}$. Além disso, a maioria das alterações evidenciadas pelos exames de rotina não contribui para alteração dos resultados, objeto do ato operatório. ${ }^{2}$ Ademais, sabe-se que um percentual significativo, equivalente a mais da metade dos resultados dos testes de laboratório, não é verificado pelos solicitantes responsáveis ${ }^{5,6}$.

Todavia, há o lado mais perverso dessa questão que são as complicações associadas ao eventual aprofundamento de investigações por causa de achados ocasionais decorrentes de exames de rotina que excederam o indicado pela avaliação clínica. ${ }^{1,6}$ Estes excessos, independente de ter sua origem ligada aos interesses pecuniários, aumentam com o conseqüente envolvimento de maior número de profissionais.

Para que isso não ocorra e o paciente fique protegido de maus resultados, a avaliação deve, principalmente, ser do "médico do paciente" com os critérios que serão discutidos, ao longo desse manuscrito.

A responsabilidade recai sobre aquele com o qual o doente tem o maior tempo de convivência e por quem o tratamento final será definido. Por este é que deve ser feita a definição dos exames de laboratórios que serão seletivos e justificados pelos achados específicos, obtidos com a história clínica e exame físico completos ${ }^{3}$.

O médico não deve ater-se a meros questionários programados, em primeiro e rápido encontro, quando respostas simples às perguntas singulares são marcadas como num teste de múltipla escolha.

Na história e exame clínico detidos, melhor do que nos exames laboratoriais e testes cardiopulmonares, estão centrados os mais importantes componentes da avaliação pré-operatória ${ }^{3}$ desde que, 
diga-se de passagem, não se confunda a avaliação préoperatória com avaliação pré-anestésica. Esta está contida naquela e clama, é óbvio, pela presença do médico anestesiologista que, na sua apreciação, deve restringir-se à área de especialização, tanto quanto lhe permite o conhecimento, e proporcionar um processo efetivo e seguro para o paciente e confortável para a equipe.

Quaisquer dispositivos contrários a essas teses estarão em descompasso com os atuais conhecimentos da prática médica; serão frutos ou do despreparo, ou podem estar apenas de acordo com os interesses mercantilistas dos que financiam a Medicina.

Há muito tempo, a Saúde vem sendo ameaçada pela colonização empresarial do médico que, iniciada dentro da Universidade, se prolonga no ambiente de trabalho. Esta ação tem origem na propaganda voraz e no ensino defasado da realidade científica. Se não for assim, origina-se na asserção de tendências individuais expressando opiniões isoladas e não abalizadas.

Pode ser, também, pela extensão de cunho popular, como influência dos atos educativos sobre as pessoas que aprendem a buscar por intervenções que visam tratar problemas somáticos ("terapêuticas ensinadas"). Essas influências provocam a mesma intensidade de desejo que demonstra qualquer outro tipo ou espécie de consumidor em relação a um determinado bem de consumo. Exames de laboratórios para alguns médicos e para os pacientes, de um modo geral, são bens de consumo.

Não é raro o paciente que, no primeiro contato com o médico, antes da apresentação da história clínica, manifesta-se, de forma veemente, com o desejo de "eu gostaria de fazer uma tomografia do abdômen". Quando não é assim, o paciente interpretando seus sintomas, antes mesmo de explicitá-los, abre ao médico um leque de sugestões de exames.

O profissional da saúde é surpreendido e falta com o decoro; descontraído, confirma os atos, principalmente na eventual situação de ser o dono dos aparelhos. Em geral ele está bem preparado para o mercado de consumo e muito mal preparado para o exercício da Medicina.

Outras vezes, o médico é o proponente - neste caso ele acena com um tipo de tratamento ou intervenção, sem deixar alternativas ao paciente. Exemplo claro é o parto por meio da cesariana, ao termo da gestação, depois do longo período de preparo feito durante os cuidados pré-natais, quando o ato cirúrgico foi colocado como o procedimento mais vantajoso. A pretensa "vantagem" recai como ônus para o paciente e para o recém-nascido, além de onerar o sistema de saúde.

Há cerca de 20 anos atrás, o Brasil assumia o primeiro lugar no mundo em número de cesarianas/ ano, que correspondia a $31 \%$ de todos os partos hospitalares. Os custos financeiros de cesarianas desnecessárias atingiam a cifra de 60 milhões de dólares anuais, e apesar das campanhas, dez anos depois, no Estado de São Paulo, 47\% dos partos eram por cesárea, independente dos custos e riscos elevados. ${ }^{7}$

Essas cenas tendem a aumentar porque o cidadão leigo, cada vez mais, passa a ter acesso, indiscriminadamente, às informações que, nem sempre, são de propriedade dos médicos; em geral excedentes, sem condições de treinar e suplementar seus conhecimentos numa Residência Médica de boa qualidade.

A antecipada visão desse fato, com tomada de consciência, foi vista na China por meio de fechamento de Escolas Médicas, como uma das diretrizes da Revolução Cultural naquele país.

No Brasil ocorreu e ocorre justamente o contrário. Com auxílio de legisladores, muitos dos quais são médicos, a criação de novas Escolas Médicas assume um caráter mercantilista, infelizmente com anuência do Ministério da Educação, apesar dos esforços contrários da Associação Médica Brasileira e do Conselho Federal de Medicina ${ }^{8}$.

A inépcia dos nossos governantes, o comprovado domínio industrial da Medicina, a mercantilização do ato médico, isto é: visar o lucro acima de qualquer outro direito e a expropriação do bem-estar vêm transformando o médico em tratador de pacientes e fomentador do consumismo na área da saúde ${ }^{1}$. Aqui, associam-se médicos e pacientes, com estreito nível de diferenciação a separar uns dos outros.

O campo de ação das Ciências Médicas passa a ser terreno de reparo e manutenção destinada a manter a máquina funcionando. O Homem, no caso, é usado como produto não humano preparado para esse tipo de consumo, no contexto do qual está inserido o médico. O médico estará mais inserido quanto menor foi seu preparo para o exercício da profissão; mão de obra barata que é, produzido pelo Estado, atual promotor 
de trabalhadores para o sistema de saúde idealizado e implantado após a década de 70, no século passado.

Por causa disso, vemos hoje repentino surgimento do que se pode chamar de "produção de saúde", sem a melhoria das condições gerais da qualidade da vida.

O que na realidade ocorre é apenas a mudança da peça alvo.

Houve enorme redução nos setores primários e secundários de ação da Saúde Pública. Ao lado disso, apareceu uma enorme tendência de inflação do terciário investigativo e terapêutico, com procedimentos complexos e máquinas altamente sofisticadas numa hábil ação de transferência, de tal modo que médicos, de um lado, tornaram-se grandes geradores do consumo e agentes mercantilistas associados; do outro, os pacientes - grandes consumidores - todos tentando ver nessa transferência um grande progresso tecnológico de aplicação social; o que, de fato, não ocorre.

Na realidade são rituais extremamente simples que alimentam o mito de uma pretensa eficácia médica. Por exemplo, qualquer sintoma abdominal (o abdômen é uma caixa de segredo!?) motiva, intempestivamente, o exame tomográfico ou ultra-sonográfico, quando não os endoscópicos de mesmas direções, mas com sentidos contrários - inúteis na maioria das vezes. A história, o exame clínico e o tirocínio do médico, num caso assim, podem determinar o diagnóstico, e o paciente receber, corretamente, tratamento para giardíase.

Outros grandes quadros poderiam ser extraídos de estudos em que se juntaria todo o progresso e implementos de técnicas modernas; de produção bioquímica e farmacêutica e de desenvolvimento biomédico, ocorridos nos últimos anos, para quantificar a transformação obtida no perfil de desenvolvimento de vários tipos de doenças ou cânceres, por exemplo. Poder-se-ia, também, verificar o que foi acrescentado em termos de conforto para o paciente canceroso.

É bem provável que o resultado não seja o que esperamos com a observada magnitude do progresso. Certamente, veríamos pacientes, com nódulos hepáticos metastáticos de adenocarcinoma de intestino grosso, não operado, recebendo longos coquetéis quimioterápicos de última geração.

Ainda que esse seja um modelo gerador de dilema clínico, isto é, qualquer circunstância nos oferecerá saídas contraditórias ${ }^{9-11}$, caberiam as seguintes questões: qual o aspecto moral, ético ou filosófico que nortearia essa conduta? Qual o impacto desse tipo de terapêutica na sobrevida?

São vários os trabalhos sobre esse assunto, mas apenas com imagens virtuais pelo número pequeno de pacientes envolvidos ${ }^{9-14}$. Setenta e dois por cento de 9.000 pacientes em condições semelhantes, nos EUA, receberam tratamento cirúrgico primário ${ }^{11}$.

No pano de fundo, persiste a influência perniciosa da indústria e da empresa médica sobre o médico. Essa influência desejável em alguns poucos aspectos é excedente em tantos outros. Tão excessiva que aqueles profissionais e os que se responsabilizam pela saúde pública consideram, hoje, irresponsável a idéia da promoção da saúde pela progressiva redução dos gastos da medicina e a sensata descaracterização de muitos cuidados médicos que poderiam ser transferidos para o campo de atuação de profissionais coadjuvantes. Isso, sem comprometer a identidade do médico e sem caracterizar o ilícito da invasão da sua área de atuação. Ë necessário que a postura profissional fuja desse quadro com a consciência de que é possível unir qualidade médica ao custo acessível. ${ }^{15}$

Reforça essas idéias o que pode ser visto nos seguintes níveis, no dizer de Illich ${ }^{1}$ :

1. As sociedades que possuem sistemas médicos muito caros são impotentes para aumentar a esperança de vida, salvo na fase perinatal;

2. O conjunto dos atos médicos tem sito incapaz de reduzir a morbidade global;

3. Atos médicos e programas de ação sanitária resultaram em fontes de novas doenças - as iatrogênicas;

4. As medidas tomadas para neutralizar a iatrogenia - outra via de consumo de produto para suportar nova ação médica - têm um efeito paradoxal, ou seja, o de tornar a doença incurável.

Ao invés da exposição desses 4 itens, poderse-ia dizer que a perniciosa influência industrial e mercantilista chegou a ponto de "criar" o médico que profere a seguinte expressão: - "Peço vários exames com o objetivo de me proteger contra uma possível condenação num questionamento legal feito pelo paciente ou pelos familiares do paciente", contra o ato médico na vigência de complicações inerentes ao próprio ato. Deixam as bases das evidências e os conhecimentos fundamentados na pesquisa científica, para praticar a teorização fundada no subjetivismo. 
Se o médico chegou a esse ponto - se de fato é um médico - alguma coisa está errada; muitas coisas estão erradas e precisam ser revistas, ou precisamos fazer a revisão do médico.

Aspecto intrigante do desenvolvimento da Medicina diz respeito à evolução do mercado paralelo que produz bens de saúde, modificações de conceitos, interpretações das doenças, meios de diagnósticos e redirecionamento das terapêuticas.

Intrigante porque as propostas oriundas de avanços industriais e os conseqüentes surgimentos de técnicas novas e complexas com potencialidades para aplicação médica, quase sempre se antepõem e são usadas antes da verificação e da consagração das novidades oferecidas. São várias as drogas lançadas no mercado com potencialidades revolucionárias no tocante aos seus efeitos sobre determinados tipos de doenças e que são rapidamente retiradas pelos próprios fabricantes. Se não tem sido assim, há as mudanças posológicas em que a dose inicial preconizada é triplicada, ou o alvo do produto - sua indicação terapêutica - é desviado do propósito inicial.

Não estamos incluindo os estudos encomendados e as promessas terapêuticas revolucionárias, fomentados por meios pseudocientíficos.

Tem sido assim, ao longo dos últimos dois séculos, principalmente após a Segunda Grande Guerra e, mais recentemente, impulsionada pela agilidade da propaganda e da hábil defesa dos interesses industriais; das inspiradas infiltrações nos centros universitários de pesquisas, do afogamento do médico como usuário ou mediador do usuário ou do consumidor e, sobretudo, da preparação dos consumidores empresariais e dos individuais da saúde. Isso é chamado de consumismo da saúde.

Seria de se supor lícitos os interesses mercantilistas, se o mundo em que vivemos não fosse tão conturbado pela corrupção do direito, da ética e da moral; mundo em que o apego ao vantajoso norteado pelo poder econômico supera aqueles de simples plasticidade, mas que são incapazes de ampliar o ganho de capital de forma impetuosa para satisfazer a ganância. Na realidade, a grande maioria de novas técnicas redefiniu as morbidades dos mais diferentes padrões de doenças, sem, no entanto, reduzí-las. ${ }^{1}$

Grandes aparelhos, procedimentos invasivos e complexos trouxeram a ilusão de correlação entre a intensidade do ato médico e o aumento da freqüência da cura. São poucas as situações em que, comprovadamente, o conjunto de métodos diagnósticos e terapêuticos sofisticados, recentemente introduzidos na prática médica, se revelou de eficácia superior aos dos procedimentos tradicionais. As Unidades de Terapia Intensiva estão lotadas - de pacientes pendurados, mais mortos do que vivos, em aparelhos sofisticados, vítimas da tentativa do prolongamento de uma vida, sem esperança de vida.

Há, no entanto, o contrário: Christian Albert Theodor Billroth introduziu, em 1861 duas técnicas operatórias que sobreviveram até os dias atuais, recebendo complicadas modificações: a primeira, a vagotomia troncular e, as outras, a vagotomia seletiva e a vagotomia superseletiva. Pretensas aliviadoras das complicações da proposta de Billroth, no tratamento das úlceras pépticas, foram recentemente trocadas com indiscutíveis vantagens pela cimetidina e os modernos bloqueadores da secreção ácido-péptica, que as sucederam, associados ou não aos antibióticos. Gastrectomia, a dois terços, reconstruída a BI ou BII, hoje soa como uma heresia. A maioria das amputações abdômino-perineais para tratamento cirúrgico do câncer do reto (operação de Miles) tem sido brilhantemente substituída pela excisão total do mesorreto com a preservação do ânus e do complexo esfincteriano, até que a cura do câncer sobrevenha pelos passos intervencionistas da engenharia genética e torne o moderno procedimento cirúrgico, também, obsoleto. Tratar a úlcera péptica ou o câncer do intestino grosso, com tamanhas mutilações, parece como "amputar o braço" por ser a pessoa hipertensa.

As outras "heréticas" são a recém introduzida operação bariátrica e a proctocolectomia total com anastomose de bolsa ileal ao ânus. A primeira para tratar a obesidade e a segunda para tratar a colite ulcerativa, em que pese o fato de que, desde suas criações - uma no final da década de 70 e a outra, nos anos 80 - os pacientes operados têm sido beneficiados, tal como ocorreu, no passado, com os ulcerosos e com os que tinham câncer do reto; alguns sobreviviam por espaço de tempo variável e a maioria morria da doença, todos com colostomia definitiva.

São os caminhos tortos da "medicina" que acabam gerando desconfiança, pelo caráter empresarial e medicamentoso que podem, eventualmente, assumir. 


\section{Exames de rotina na avaliação pré-operatória}

A introdução teve a finalidade de despertar atenção para os objetivos do tema proposto, lembrando que a chave central ou o maior propósito é, também, provocar discussão em torno da seguinte afirmação: "quanto mais se exige para um ato uma infra-estrutura dispendiosa, maiores são as probabilidades de que"1:

1. a esperança de sobrevida do paciente submetido ao ato não seja modificada pelo ato;

2. o período de invalidez do paciente aumentará, e

3. o paciente terá necessidade de tratamento adicional para ajudá-lo a suportar os danos, mutilações, angústias e dores provocadas pela intervenção.

É óbvio que, com certeza, eles não se aplicam aos exames pré-operatórios de rotina, mas ao quanto de negligência que pode estar associada à falsa sensação de proteção sentida pelo médico, quando ele cerca seus pacientes com os referidos exames ou quando resultados inesperados desviam o médico de seu objetivo primordial.

Assim, essas coisas menores acabam seguindo aqueles postulados e dentro dessas menores vamos falar muito rapidamente sobre a rotina desnecessária de exames pré-operatórios, para depois nos determos à avaliação cardiovascular pré-operatória em pacientes que serão submetidos a operações não cardíacas.

As "rotinas" desnecessárias serão agrupadas supondo que os exames são escolhidos por propósito de melhor avaliação do paciente, sem o respaldo da História Clínica. Assim vamos agrupá-los dentro dos 6 itens que são:
A. Função cardiorespiratória e transporte
B. Função imunológica e infecção
C. Função renal
D. Função hepática e coagulação
E. Função endócrino-metabólica e eletrolítica
F. Reposição volêmica ou prática transfusional

Esses podem ser mais especificados assim:

A. Função cardiorespiratória e transporte Os exames são - hemograma completo (hematócrito e hemoglobina), raios X de tórax e eletrocardiograma nas 12 derivações clássicas. Espirometria.

B. Função imunológica e infecção Os exames são: leucograma e perfil associado, e urina tipo I

C. Função renal

Os exames são: urina tipo I, uréia e creatinina.

D. Função hepática e coagulação

Os exames são: enzimas hepáticas, tempo de protrombina, plaquetas e coagulograma.

E. Função endócrino-metabólica e eletrolítica

Os exames são: glicemia e eletrólitos.

F. Reposição volêmica ou prática transfusional

Os exames são: eritrograma, tipo sangüíneo (sistema ABO e Rh) e contra prova, proteínas totais e frações.

Nessa classificação separamos os seguintes 17 ou mais exames (Tabela-1.)

Os exames ditos de rotina têm recebido críticas baseadas em estudos racionais sobre a avaliação préoperatória $^{2,3}$ de tal modo que, exceto para os idosos, dificilmente iríamos conseguir, com base em critérios clínicos e fundamentados nos conhecimentos atuais, pedir mais que 3 exames para um paciente, na véspera da operação ${ }^{3}$. Coelho e col. ${ }^{2}$ exploraram de forma simples e concisa esse assunto, o que recomenda a leitura daquele artigo de atualização.

Já houve época em que se estabelecia um conjunto de exames laboratoriais pré-operatórios que envolvia, nada mais nada menos, 21 exames, dos quais 17 estão listados na Tabela-1. Mais tarde, tornaram-se

Tabela 1 - Exames laboratoriais mais comumente pedidos, de maneira inespecífica, como rotina de avaliação pré-operatória.

\begin{tabular}{llll}
\hline 1.Hemograma & 5. Sódio, 6. Potássio & 12.Coagulograma & 16. Tipo de sangue \\
2.Urina tipo I & 7. Proteínas & 13. Glicemia & 17. Contra prova \\
3.Uréia & 8. Bilirrubinas & 14. Rx Tórax & 18. e outros \\
4.Creatinina & 9.TGO,10.TGP, 11.TP & 15. ECG &
\end{tabular}


obsoletos pela inutilidade, por ser mais causadores de confusão do que esclarecedores, além de muito caros ${ }^{3}$.

O que se observou foi que quanto mais exames eram solicitados, maior era o número de resultados anormais. Testes laboratoriais feitos em pessoas normais darão resultados anormais em $5 \%$ das vezes e, quando 10 exames são feitos, há $50 \%$ de probabilidade que um dê resultado anormal. ${ }^{16}$

Por outro lado, os resultados anormais inesperados, mas observados na maioria dos exames laboratoriais, não provocam mudança na conduta seguinte que é o ato operatório. Isso tem sido válido para valor de hemoglobina, tempo de sangramento, tempo de protrombina, tempo de coagulação, tempo da tromboplastina ativada, sódio, potássio, urina tipo I, raios-X de tórax e eletrocardiograma.

Estamos de volta àquela época. Não nos limitando exclusivamente ao pré-operatório, diríamos que a demanda crescente no número de exames solicitados para conduzir, antes de confirmar, ao diagnóstico, tem, sem atingir a pretendida qualidade, aumentado assustadoramente o preço dos cuidados médicos. Nem cogitamos considerar que quem não sabe o que procura, em geral, não interpreta o que acha.

Aliado a isso, a imposição ilusória de salvaguardas, com baterias de exames, tem se tornado em ponto conflitante entre profissionais de diferentes áreas por causa da atitude antiética e do ato comisso. Esse, contrário ao da omissão, é o erro que se comete quando se faz o que pode resultar em prejuízo. Ambos, a omissão e o ato da comissão contemplam a imprudência; são erros comuns e um tão grave quanto o outro.

Um ato comisso pode ser observado na situação em que um nódulo de pulmão, que foi encontrado por meio de exame radiológico de tórax desnecessariamente solicitado na avaliação préoperatória de um paciente, motiva a reorientação de conduta. Esse achado desnecessário foi a razão para o aprofundamento da investigação. Biopsias transparietais, toracotomia, iatrogenias e complicações graves decorrentes foram as sucessivas ocorrências. A morte do paciente foi o resultado final, sem que ele sequer tivesse sido tratado do motivo pelo qual procurou o médico. O nódulo não era expressão de lesão que justificasse a investigação e não interferiria com o ato cirúrgico proposto.
O outro ato comisso é a punção da jugular ou da subclávia, quando a punção de veia periférica satisfaz plenamente as razões para o acesso venoso.

O exame desnecessário, a interpretação equivocada, o ato comisso, a incontestável imprudência podem ser os motivos para o desfecho fatal. Fatos como esses, em geral, são originados pelo "marketing" do médico ou da empresa.

Não se mede esforço para a proteção do paciente; mas exames, pelo simples fato de ser, não significam esforço; não protejem o paciente, muito menos o profissional; não qualificam o atendimento, pelo contrário, podem desqualificar, criando um perfil indesejável da doença, do paciente e do médico.

A avaliação pré-operatória com esforço de qualificar o atendimento e atingir resultados seguros é tarefa exeqüível e exigente de ação primária, mas conturba o médico, conduzindo-o ao ato anacrônico da chamada Medicina defensiva, grande aliada da medicamentação e mercantilização da saúde, principalmente a que recomenda o cerco do paciente com os exames para acobertar outras deficiências.

Num grupo de 400 pacientes com doenças colorretais, a maioria dos quais foi submetida a operações abdominais de médio e grande porte, encontramos 250 deles com doenças associadas: - 29 pacientes $(11,6 \%)$ com três moléstias; 70 (28\%), com duas e 138 (55\%) com uma.

Os exames de rotina, nesse grupo, não excederam aqueles feitos para os outros 150 pacientes portadores apenas da moléstia alvo do tratamento cirúrgico.

Os procedimentos operatórios foram desenvolvidos com técnicas clássicas de acordo com a exigência da doença cirúrgica. As complicações observadas não foram, em número nem em gravidade, diferentes das observadas no grupo de pacientes mais saudáveis. Considerando os fatores de risco avaliados para aqueles grupos, os exames obtidos no préoperatório não foram orientadores de condutas de salvaguardas capazes de influenciar os resultados ${ }^{17}$.

Para pacientes hígidos (os portadores apenas da doença cirúrgica), dificilmente seríamos capazes de justificar exames laboratoriais. Para os pacientes com doenças associadas, os exames devem ser limitados às exigências de cada doença avaliada.

Por outro lado, a dosagem da creatinina sérica deve fazer parte da rotina para os pacientes com idade 
superior a 50 anos, faixa etária em que os valores podem estar iguais ou acima de $2 \mathrm{mg} \%$, refletindo a insuficiência renal assintomática, relativamente comum acima dessa idade ${ }^{16,18}$. Nesses casos, o conhecimento do fato poderá orientar o ajuste posológico de drogas, eventualmente usadas no peroperatório.

Dentro desse contexto, no entanto, há um grupo especial de doentes que tem sido alvo de divergências quando se discute entre médicos (clínicos, cirurgiões e anestesistas) sobre a avaliação préoperatória com ênfase aos cuidados exigidos, necessários e suficientes, para o bom desempenho do tratamento. São os pacientes cardiopatas, candidatos a operações no âmbito de qualquer especialidade cirúrgica, exceto a cardíaca.

As divergências são originadas mais pela falta de critério do que por critérios embasados em fundamentos científicos ou em desvelos. A falta de critério mencionada decorre mais da propaganda que se desenvolve paralela ao progresso da Medicina, fato objeto desse manuscrito, do que do conhecimento de ajustes e cuidados proporcionados pelos avanços modernos e pelo equilíbrio do exercício profissional.

Os pacientes cardiopatas, então reconhecidos, tornam-se alvos da discórdia, quando alguns querem enquadrá-los num grupo de risco de classificação imprópria e então exigir exames, procedimentos e cuidados pré-operatórios, incapazes de desviar o curso do processo ou garantir o melhor resultado, quando não põem em risco a integridade do paciente.

\section{A. Avaliando clinicamente o paciente cirúrgico cardiopata}

Ainda aqui, a história clínica, o exame físico, a identificação da doença cardiovascular associada quanto às suas características, gravidade, tempo de evolução, tratamento anterior e repercussão funcional são demandas essenciais. Mais do que qualquer tipo de exame ou de testes, são os recursos seguros para orientar condutas com o propósito de se evitar testes complexos e caros, às vezes invasivos e associados a riscos, que certamente não vão corresponder com a expectativa de maior proteção contra as possíveis complicações decorrentes do ato operatório.

A avaliação desses pacientes pode cuidadosamente se desenvolver num plano que contemple os objetivos primários, desde que sejam delineados dentro de um contexto vinculado à natureza da moléstia, motivo da operação, e às características do ato cirúrgico, em si. Nesse aspecto, é importante lembrar que a condução dependerá do caráter do ato cirúrgico que pode ser eletivo, urgente ou emergente. Além disso, a equipe deve participar de forma integral, usando, não só os mesmos meios de comunicação, como também os do entendimento. Devem lembrar que os eventuais exames usados na avaliação pré-operatória de pacientes cardiopatas candidatos às operações de outros órgãos são os mesmos, tais quais os que seriam usados na avaliação cardiológica dos mesmos doentes se eles estivessem fora da situação em que estão sendo apresentados agora.

Então, complementado o que foi dito alguns parágrafos acima, o ECG e o questionário embasado no exame físico especial serão dirigidos para identificar o potencial mórbido da doença cardíaca, inclusive a coronariopatia; história precedente de infarto do miocárdio, dor precordial anginosa, debilidade funcional do miocárdio (grau de insuficiência cardíaca), arritmias sintomáticas, a presença ou não de marcapasso e concomitância de anemia, que pode e deve ser avaliada por meio de eritrograma, são os itens norteadores da avaliação.

Repete-se que não se deve interessar em apenas identificar a doença, mas em graduá-la quanto a sua gravidade, sob todos os aspectos clínicos. Não se pode esquecer que muitos desses pacientes têm outras doenças associadas, tais como diabetes, doenças renais, doenças vasculares periféricas ou pulmonares, e são idosos.

A capacidade funcional dessas pessoas deve e pode ser facilmente aquilatada sem a necessidade de dispensar-lhes fórmulas ou exames de alta complexidade.

Essa avaliação, complemento do exame físico especial, adquire maior relevância quando estamos, para completar a definição do risco operatório, associando o risco cardíaco aos fatores agravantes exclusivos do porte cirúrgico a que será submetido o paciente. Todavia, antes dessa tarefa é melhor definir os testes pré-operatórios de avaliação de riscos e eleger os pacientes que, submetidos aos testes, poderão ser tratados e, consequentemente, beneficiados. Há uma grande variedade de caminhos para esse intento, mas não há nada que defina a melhor estratégia. 
Não deixa de ter algum valor, dentro do tipo de raciocínio até então desenvolvido, destacar um exame que tem sido intocável quando se fala em avaliação pré-operatória. Trata-se do eletrocardiograma.

O eletrocardiograma de repouso tem sido exigido sistematicamente como fator de avaliação indispensável, para o que, invariavelmente, se dá desmedida ênfase e papel de inválida representação. Esse exame não aumenta a identificação de risco cirúrgico, em operações de baixo risco, mas auxilia nas situações em que se trata de doentes classificados entre aqueles com marcadores clínicos graves ou intermediários. Assim, como instrumento de mensuração de maior ou menor segurança, o ECG somente estará indicado para pacientes com história pregressa de infarto do miocárdio (IM), episódios recentes de precordialgia ou para os outros que podem estar dentro daquela classificação de risco grande ou intermediário, que serão descritos logo adiante. Nos diabéticos assintomáticos, nos pacientes com história de revascularização do miocárdio, em homens assintomáticos acima de 45 anos ou mulheres acima de 55 anos, a indicação tem merecido controvertidas disputas ${ }^{4}$. Para outras situações, acrescenta absolutamente nada ${ }^{4}$.

O Colégio Americano de Cardiologia e a Associação Americana do Coração fizeram uma proposta de consenso, em 1996, consolidada e revista em 2002, quando estabeleceram as diretrizes básicas de avaliação pré-operatória de riscos para os pacientes cardiopatas eleitos para operações não-cardíacas ${ }^{4}$.

Os fundamentos do algoritmo congregam apreciação em diferentes níveis de riscos atribuídos às diferentes circunstâncias clínicas, não só as relativas ao coração, como aos:

\section{1. marcadores clínicos de prognósticos de} riscos,

2. à capacidade funcional, bem como as que se associam, por acaso ou em decorrência da cardiopatia, além das que derivam de

3. tipo de operação proposta, quanto ao porte e à oportunidade de sua execução (emergência ou eletiva).

A cada passo, no desenvolvimento do algoritmo, dados clínicos descritos como os marcadores clínicos, a capacidade funcional e os riscos inerentes ao tipo de operação são contabilizados.

\section{Marcadores clínicos}

Os principais marcadores clínicos de prognósticos de riscos estão classificados em Graves, Moderados e Leves (Tabela-2).

As definições contidas na Tabela-2 refletem o consenso do (Colégio Americano de Cardiologia Banco de Dados do Comite Cardiovascular) ACC Cardiovascular Database Committee, com destaque para as diferentes considerações sobre o infarto do miocárdio, revogando a anterior classificação em intervalos de 3 e 6 meses após o infarto ${ }^{19,20}$, para dar ênfase a uma estratificação de riscos durante a fase de convalescença do IM, com a recomendação de que, na ausência de sintomas, deve-se esperar pelo menos 4 a 6 semanas para liberar o paciente para uma operação eletiva. $^{21}$

\section{Capacidade funcional}

A capacidade funcional, item do interrogatório sobre os diversos aparelhos e sistemas, é facilmente avaliada em termos de níveis de equivalente metabólico (EM) - Tabela-3..$^{22-24}$ Valores múltiplos do EM podem ser usados para expressar a demanda aeróbica durante exercícios específicos.

Os índices de capacidade funcional variam de 1 a 4 , acima de 4 a 10 e acima de 10 e provêm ao médico, por meio de questionário simples, um método exequíivel de avaliação da capacidade funcional do paciente.

Os riscos peroperatórios para pacientes cardiopatas submetidos a operações não cardíacas aumentam para os que não atingem índice EM-4, que expressa o gasto em atividades domésticas corriqueiras e cuidados pessoais, tais como vestir-se, alimentar-se, caminhar pela casa, ou lavar pratos; valores acima de 4 até 10 representam a capacidade para subir degraus de uma escada, subir uma ladeira, andar no plano a uma velocidade de $6 \mathrm{~km}$ por hora, escovar o chão ou correr uma curta distância. Atividades como jogar futebol, jogar uma simples de tênis e a natação excedem o valor EM-10.

\section{Riscos específicos do ato operatório}

Os riscos atribuídos ao ato cirúrgico estão relacionados a dois importantes fatores, a saber: primeiro, ao porte da operação e, segundo, ao grau dos 
Tabela 2 - Marcadores clínicos de prognósticos de risco classificados de acordo com o grau.

\begin{tabular}{|c|c|c|}
\hline & Moderado & Leve \\
\hline Grave & Angina moderada & Idade avançada \\
\hline \multicolumn{3}{|l|}{$\begin{array}{l}\text { Síndrome coronariana instável } \\
\text { · IAM com risco isquêmico importante, } \\
\text { avaliado clinicamente. }\end{array}$} \\
\hline $\begin{array}{l}\text { - Angina instável grave } \\
\text { Insuficiência cardíaca }\end{array}$ & IM prévio - onda Q anormal & $\begin{array}{l}\text { ECG anormal (hipertrofia, } \\
\text { bloqueio do ramo esquerdo e } \\
\text { anormalidade ST-T). }\end{array}$ \\
\hline & IC compensada & Fibrilação atrial \\
\hline $\begin{array}{l}\text { Arritmias significantes } \\
\text { - Bloqueio A-V de alto grau } \\
\text { - Arritmia ventricular sintomática na } \\
\text { presença de doença cardíaca } \\
\text { - Arritmia supraventricular com freqüência }\end{array}$ & & \\
\hline $\begin{array}{l}\text { ventricular incontrolável } \\
\text { Doença valvular grave }\end{array}$ & $\begin{array}{l}\text { Diabete insulina dependente } \\
\text { Insuficiência renal }\end{array}$ & $\begin{array}{l}\text { Capacidade funcional baixa } \\
\text { Historia de derrame } \\
\text { Hipertensão sistêmica } \\
\text { incontrolada }\end{array}$ \\
\hline
\end{tabular}

IAM=infarto agudo do miocárdio, IM=infarto miocárdio, ECG=eletrocardiograma, IC=insuficiência cardíaca

Tabela 3 - Valores estimados de energia no desenvolvimento de várias atividades ${ }^{22,24}$

\begin{tabular}{ll} 
EM-1 a & EM-4 a \\
EM-4 & EM-10 \\
\hline
\end{tabular}

Prover cuidados pessoais

- Alimentar-se

- Vestir-se

- Usar o banheiro

- Caminhar ao redor da casa

- Andar 1 quadra ou 2 à velocidade de $4 \mathrm{~km} / \mathrm{h}$

EM-4

- Remover sujeiras

- Limpar ou lavar pratos
EM-10

Afazeres domésticos leves

- Subir um lance de escada; subir ladeira

- Andar no plano á velocidade de $6 \mathrm{~km} / \mathrm{h}$

- Correr uma curta distancia

- Afazeres domésticos mais pesados: varrer e escovar o chão, mudar moveis de lugar

- Recreações: dançar, golfe, dupla de tênis

Exercícios extenuantes

- jogar futebol

- natação

- $\quad$ simples de tênis

- basquete

- esquiar 
distúrbios metabólicos inerentes ao procedimento, não só em termos de desequilíbrio hemodinâmico que decorrem daqueles distúrbios, mas a todas as alterações inerentes ao cenário em que se incluem a internação, o ambiente hospitalar, o jejum pré-operatório, a anestesia e o ato cirúrgico em si. Este pode ser classificado, quanto ao risco, em operação de grande, médio e pequeno risco.

As operações de maiores riscos incluem as de emergência, que não são alvos dessa revisão; as operações aórticas, as grandes operações vasculares periféricas e as operações de grande porte reconhecidamente longas, nas quais incidem trocas consideráveis de líquidos entre os compartimentos orgânicos e naquelas em que se espera uma maior perda sanguínea. As outras estão explicitadas na Tabela-4.

Definidos os critérios de riscos 1,2 e 3 (marcadores clínicos, capacidade funcional e tipo de operação) passa-se por 8 etapas, bem estabelecidas em termos de definição clínica em que os aspectos principalmente destacados são:

a. urgência do procedimento cirúrgico proposto,

b. presença ou não de doença arterial coronariana (DAC), já diagnosticada;

c. capacidade funcional do coração presença ou não de insuficiência cardíaca (IC) e,

d. doença valvular grave.

As oito etapas mencionadas contemplam todos os marcadores clínicos de prognóstico (grave, moderado ou leve) explicitados na Tabela-2, bem como os demais indicadores de riscos. ${ }^{3,4}$
Nas etapas de 1 a 8, armadas num algoritmo, as questões são simples quando as respostas previsíveis orientam o passo seguinte cuja definição sempre busca por procedimentos não invasivos e por terapêuticas clínicas de baixo custo e alta efetividade. ${ }^{4}$

\section{B. Identificação de condições cardiovasculares patológicas específicas e seu manuseio}

\section{a. Hipertensão arterial}

A hipertensão arterial (sistólica acima de 180 $\mathrm{mmHg}$ e diastólica igual ou superior a $110 \mathrm{mmHg}$ ) deve ser identificada, eventualmente estudada e controlada antes da programação da operação eletiva, podendo a intervenção ser adiada por dias ou semanas, até que se estabeleça o controle efetivo dos níveis pressóricos. Nos casos de urgência ou emergência, as considerações serão diferentes e, salvo em opiniões contrárias impostas por avaliação precisa, os beta-bloqueadores recebem destaques, como os agentes mais apropriados para o controle rápido da pressão arterial, devendo ser mantidos ao longo do período pós-operatório..$^{25-30}$

b. Doenças valvulares

A avaliação dessa condição não foge, aqui, daquilo que está determinado para qualquer portador de valvulopatia, vivendo fora do cenário de uma operação cirúrgica. As lesões valvulares estenóticas são fatores causais relevantes da insuficiência cardíaca aguda peroperatória. Elas, sob juízo do cirurgião cardiotorácico, podem ser alvo de intervenção corretiva que visam colocar o paciente para a operação não-cardíaca em níveis do mais baixo risco ${ }^{19,31,32}$

\section{c. Doenças do miocárdio}

A dilatação do coração e a hipertrofia do miocárdio estão associadas com falência funcional do

Tabela 4 - Classificação do risco cardíaco $(R C)$ de acordo com o porte e tipo de procedimento cirúrgico.

\begin{tabular}{|c|c|c|c|}
\hline $\begin{array}{ll} & \text { Porte } \\
\text { Tipos } & \end{array}$ & Grande $(\mathrm{RC}>5 \%)$ & Médio $(\mathrm{RC}<5 \%)$ & Pequeno $(\mathrm{RC}<1 \%)$ \\
\hline Eletiva & $\begin{array}{l}\text { Urgência no idoso } \\
\text { Aorta }\end{array}$ & $\begin{array}{l}\text { Endarterectomia carotídea } \\
\text { Cabeça e pescoço }\end{array}$ & $\begin{array}{l}\text { Procedi/to } \\
\text { endoscópico }\end{array}$ \\
\hline ou & Vascular periférica & Abdominal, torácica & Hérnias \\
\hline Urgência & $\begin{array}{l}\text { Operação demorada c/ } \\
\text { sangramento } \\
\text { Amputação do reto }\end{array}$ & $\begin{array}{l}\text { Ortopédica } \\
\text { Prostática }\end{array}$ & $\begin{array}{l}\text { Vesícula } \\
\text { Mama } \\
\text { Catarata }\end{array}$ \\
\hline
\end{tabular}


coração, no peroperatório $^{19}$. O grau de hipertrofia ou a dilatação cardíaca exigem avaliação acurada e tratamento adequado antes da submissão ao ato cirúrgico. Os objetivos, em reconhecendo esses estados, são providenciar a avaliação da reserva hemodinâmica e preparar a monitorização e terapêutica continuada no pós-operatório.

\section{d. Arritmias e distúrbios de condução}

Esses são dois dados que devem chamar a atenção para diagnóstico etiológico ou associação com doenças pulmonares, estado tóxico metabólico ou com intoxicação medicamentosa.

A terapêutica deve ser iniciada para os pacientes sintomáticos ou para os que têm arritmias com estabilidade hemodinâmica, a princípio para reverter a causa e depois para tratar a arritmia sintomática. Dessa forma as indicações de intervenção clínica são as mesmas para os pacientes, nas mesmas circunstâncias, mas que não vão ser submetidos à intervenção cirúrgica. As extra-sístoles ventriculares e as taquicardias ventriculares não sustentadas, assintomáticas, não estão relacionadas a complicações maiores no pós-operatório, nem com o maior risco de IM ou morte cardíaca, razão por que qualquer estudo aprofundado e medicação agressiva, nesses casos, devem ser dispensados ${ }^{4}$

\section{Avaliação suplementar}

Ficou claro que, em se tratando de pacientes cirúrgicos com cardiopatias, as medidas de preocupação no pré-operatório são individualizadas e orientadas de acordo com cada paciente e a situação envolvida.

Os itens seguintes podem ser os componentes apropriados de uma avaliação dirigida e serão rapidamente mencionados com descrição sumária de cada circunstância que indica sua necessidade. São eles:

1. avaliação, em repouso, da função ventricular

2. monitorização eletrocardiográfica ambulatorial *

3. teste de estresse ou teste farmacológico durante o estresse

*Na maioria dos pacientes a escolha é para o teste especificado no item $b$, pela propriedade que tem a monitorização eletrocardiográfica em exercício, tanto em fornecer dados estimativos da capacidade funcional como também detectar alterações isquêmicas do miocárdio por meio de alterações gráficas e das repostas hemodinâmicas.
As recomendações para os testes acima mencionados são as seguintes:

\section{Função ventricular no repouso:}

A avaliação da função do ventrículo esquerdo, no repouso, não tem valor para previsão de isquemia miocárdica peroperatória ${ }^{33,34}$. Está indicado, porém, para pacientes com diagnóstico clínico de insuficiência cardíaca ou insuficiência cardíaca de difícil controle ou compensação. Esse teste não deve, portanto, fazer pare de rotina para os cardiopatas que irão ser submetidos às operações de outros órgãos.

\section{Eletrocardiograma}

Comentamos acima a respeito do ECG, tentando desviar a atenção das funções que supostamente são destinadas ao ECG, como exame de rotina no pré-operatório. Sua indicação indiscriminada não tem lugar nem mesmo entre os pacientes que se enquadram na situação especial que ora estamos tratando. Esse exame tem indicação inquestionável para pacientes cirúrgicos com história recente de dor precordial ou isquêmica que podem ser colocados na escala de alto risco ou de risco intermediário determinado pelos marcadores clínicos ou para os que irão ser submetidos a procedimentos cirúrgicos, por si só, de alto risco para complicações cardíacas indesejáveis. Dentro do grupo das cardiopatias, as outras indicações demandam posições de conflitos por evidência ou por opinião a respeito de sua utilidade ou eficácia, com forte tendência para as conclusões de inutilidade. Essa assertiva tem ênfase quando, em se tratando de cardiopatas, nos deparamos com pacientes já submetidos, com sucesso, a revascularização do miocárdio. É válida, também, para homens ou mulheres acima dos 45 anos, por esse simples fato; para pacientes com história de uma única internação hospitalar por causa de distúrbio cardíaco e, finalmente, podemos repetir, não há respaldo para que o ECG seja incluído como exame de rotina para pacientes assintomáticos, ainda que cardiopatas, que serão submetidos a operações de baixo risco.

\section{Atividade de estresse ou ação farmacológica} em atividade de estresse

O estudo sob o efeito de exercício ou sobre o efeito farmacológico de certas drogas, sob estresse, tem indicação incontestável nas seguintes condições: 
- pacientes com suspeita de DAC

- avaliação prognóstica de pacientes com DAC

- demonstração de isquemia miocárdica antes de procedimentos de revascularização.

- avaliar a adequação e eficácia de um tratamento e avaliaão prognóstica depois da revascularização

Todos os outros motivos a serem aventados suscitarão conflitos de evidências ou de opiniões.

\section{Medicação pré-operatória - uma rotina preventiva ?}

Estima-se que cerca de 26 milhões de americanos são anualmente submetidos a operações não cardiacas $^{35,36}$ e que dentro desse grupo, 1 a 5\% são vítimas de complicações cardiovasculares peroperatórias, em geral do tipo isquêmico, com custo aproximado de 20 bilhões de dólares. ${ }^{35,37}$

As normas estabelecidas de avaliação de risco, prognósticos e condutas preventivas aplicáveis a pacientes cardiopatas candidatos a operações nãocardíacas foram geradoras de estudos sobre ação protetora de certas substâncias contra as alterações miocárdicas isquêmicas, sobretudo nos pacientes com DAC e nos hipertensos refratários. Entre esses medicamentos, o destaque tem recaído sobre os betabloqueadores, principalmente estimulado por dois autores participantes do estabelecimento daquelas normas $^{36}$, seguidos posteriormente por outros ${ }^{39-42}$, com resultados que nos orientam para a posição de observadores comedidos.

Principalmente indicados para situações como as caracterizadas pela DAC e a hipertensão arterial com a hipertrofia concêntrica do coração, tiveram o propósito de estabelecer um equilíbrio entre a demanda e a oferta de $\mathrm{O}_{2}$ para o miocárdio. $\mathrm{O}$ uso desses bloqueadores foi estendido para outras cardiopatias, tais como a insuficiência cardíaca, as doenças vasculares periféricas e para diabéticos com hipertensão $\operatorname{arterial}^{39}$.

A despeito das recomendações feitas pela "American Heart Association and American College of Cardiology" para o uso de beta-bloqueadores em pacientes com fatores de risco para DAC ou com coronariopatia diagnosticada que iriam ser submetidos a qualquer tipo de operação de alto risco (intratorácica ou intra-abdominal), as evidências de eficácia para esses propósitos, oriundas de estudos casualizados duplo-cego são raras e com resultados controversos ${ }^{43}$, tais como os que foram observados nos projetos "DECREASE" (Dutch Echocardiographic Cardiac Risk Evaluation Applying Stress Echocardiography) ${ }^{44}$, em que a proteção cardíaca foi de $90 \%$ com o uso do bisoprolol e no "DIPOM" (Diabetic Postoperative Mortality and Morbidity) $)^{45}$, em que os resultados não puderam ser confirmados, ou no estudo mais recente feito por Lindenauer e col. ${ }^{41}$, em que as observações sobre os beta-bloqueadores são evidências para que seu uso seja rigorosamente seletivo e fiquem reservados para pacientes com marcadores clínicos classificados como graves, já que a relação entre o uso pré-operatório de beta-bloqueadores de proteção e o risco de morte operatória foi inversamente relacionado com o risco cardíaco. Isto é, o beta-bloqueador foi associado com $43 \%$ de aumento no risco de morte cardíaca nos pacientes de baixo risco (índice de escore igual a 0 ; $\mathrm{RCRI}^{1}$ ) e em $13 \%$ entre os pacientes com escore igual a 1 (RCRI) ${ }^{41}$, mas, numa relação direta, protegeu os pacientes cujos escores de riscos eram altos (variando de 2, 3, 4 ou acima de 4), num índice de proteção que variou de 10 a $43 \%$.

Em suma, o que podemos subtrair de todos esses dados é que "ainda há muito poucos estudos prospectivos, casualizados e bem controlados sobre a intervenção clínica em pacientes cardiopatas que se submeterão às operações não cardíacas. Os resultados dos estudos disponíveis não são os suficientes para firmes conclusões e corretas recomendações"4,46.

Todos os testes citados, a priori, poderiam ser dispensados na avaliação pré-operatória dos pacientes cardiopatas ou não. Depreendemos que os exames da coletânea mencionada podem ser necessários, indispensáveis e até essenciais para reorientar o planejamento terapêutico. Podem ser capazes de substituir o tratamento cirúrgico de uma doença pouco grave pelo tratamento clínico de outra muito grave, mas serão indicados de acordo com os critérios embasados na história e no exame clínico do paciente, e não em "protocolos de rotina" ou "kit" de exames pré-operatórios para atender interesses alheios ao conjunto médico-paciente.

\section{${ }^{I} R C R I=$ Índice de risco cardíaco revisado}


São dados que podem parecer isolados, mas que nos remetem à proposição inicial desse manuscrito e permitem que façamos inclusão, aqui, de preocupações, já manifestas, relativas a um grande número de publicações ou porque elas são clinicamente mal conduzidas ou porque, às vezes, mais expressam o desejo comercial da valorização de um produto de aplicação clínica, do que a demonstração da sua real eficácia, em termos de custobenefício. ${ }^{47-49}$

SUMMARY: Background: The Health has been threatened by the physician's managerial colonization that, begun inside of the University, continues in the work environment. That action has origin of the teaching in disagreement with the scientific reality and in the assertion of individualistic tendencies expressing isolated and not-distinguished opinions, in general induced by the investigations requested to give rise to the market of drugs and devices disposable for physician and pharmacists.

Purpose: The aim of this report is to use the General Surgeon's point of view to comment on the commercialization of the health and on the way as the Industrial Institution, using the medical company, acts and modifies the physician's action contributing to the high cost of the medicine.

Material and Methods: The bases will be the laboratory exams and the preoperative cardiovascular routine evaluation used for noncardiac surgery on patients with cardiomyopathies. The material and the content for discussion were extracted from Ivan Illich's ${ }^{1}$ book, Coelho and col. ${ }^{2}$ article, from the model proposed in Cleveland Clinic ${ }^{3}$ on preoperative tests, and from the established norms of $\mathrm{ACC}^{1}$ and of the $\mathrm{AHA}^{*}$ about the orientation on minimum medical evaluation and exam of patient with cardiac disease, when they are candidates to a noncardiac surgery ${ }^{4}$.

${ }^{1}$ ACC $=$ American College of Cardiology; AHA= American Heart Association

Key words: Laboratory tests; preoperative evaluation; noncardiac surgery; health commercialization.

\section{REFERÊNCIAS BIBLIOGRÁFICAS}

1. Illich I. A expropriação da Saúde: Nêmesis da Medicina. $3^{\circ}$. Edição, 1975. Editora Nova Fronteira.

2. Coelho JCU, Campos JCJ, Tenório SB. Solicitação de exames pré-operatórios. Rev Col Bras Cir 1997;25:353-58.

3. Michota FA, Frost SD. The preoperative evaluation: Use the history and physical rather than routine testing. Cleveland Clin J Med 2004;71:63-70.

4. Task Force Members. ACC/AHA Guideline Update for Perioperative Cardiovascular Evaluation for Noncardiac Surgery - Executive Summary - A Report of the American College of Cardiology/American Heart Association Task Force on Practice Guidelines (Committee to Update the 1996 Guidelines on Perioperative Cardiovascular Evaluation for Noncardiac Surgery). Circulation. 2002;105:1257-67.

5. Roizen ME, Kaplan EG, Schreider BD, et al. The relative role of the history and physical examination, and laboratory testing in preoperative evaluation for outpatient surgery: the "Starling" curve of preoperative laboratory testing. Anesthesiol Clin North Am 1987;5:15-34.

6. Roizen MF. Preoperative evaluation, In Miller RD (ed): Anesthesia, $4^{\mathrm{a}}$. Ed. New York - Churchill-Levingstone, 1994;pp.827-34.

7. Word Develompent Report. Investing in Health. World Bank, Oxford University Press, 1993
8. Loures Bueno RR e Pieruccini MC.Conselho Federal de Medicina. Abertura de Escolas de Medicina no Brasil: relatório de um cenário sombrio. $2^{\mathrm{a}}$. edição, 2005

9. Sarela AI, O'Riordain DS. Rectal adenocarcinoma with liver metastases: management of the primary tumour. Br J Surg 2001;88:163- 64.

10. Sarela AI, Guthrie JA, Seymour MT, et al.. Non-operative management of the primary tumour in patients with incurable stage IV colorectal cancer. Br J Surg 2001; 88: 1352-56.

11. Temple LK, Hsieh L, Wong WD et al.. Use of surgery among elderly patients with stage IV colorectal. cancer. J Clin Oncol 2004; 22: 3475-84.

12. Tebbutt NC, Norman AR, Cunningham D, et al. Intestinal complications after chemotherapy for patients with unresected primary colorectal cancer and synchronous metastases. Gut 2003; 52: 568-73.

13. Benoist S, Pautrat K, Mitry E, et al. Treatment strategy for patients with colorectal cancer and synchronous irresectable liver metastases. Br J Surg 2005; 92: 1155-60.

14. Lemmens V E P P, Janssen-Heijnen M L G, Verheij C DG W, et al. Co-morbidity leads to altered treatment and worse survival of elderly patients with colorectal cancer. Br J Surg 2005; 92: 615-23.

15. Zucchi P, del Nero C, Malik AM. Gastos em Saúde: os fatores que agem na demanda e na oferta dos serviços de saúde. Saúde e Sociedade 2000;9: 127-50. 
16. MacPherson DS: Preoperative laboratory testing: should any tests be 'routine' before surgery? Med Clin North Am 1993;77:289-308.

17. Santos Jr. JCM. Fatores de riscos associados às complicações cirúrgicas em operações de resseccão e anastomose do intestino grosso sem o preparo mecânico - estudo da incidência de infecção e deiscência da anastomose. Rev bras Coloproctol 2005.25:168-87.

18. Roizen MF. Preoperative evaluation. In R D Miller (ed). Anesthesia - 4 ${ }^{\text {a }}$. Edition New York Churchil-Livingstone, 1994;pp 827-34.

19. Goldman L, Caldera DL, Nussbaum SR, et al. Multifactorial index of cardiac risks in noncardiac surgical procedures. N Engl J Med 1977;297:845-50.

20. Tarhan S, Moffit EN, Taylor WF, et al. Myocardial infarction after general anesthesia. JAMA 1972;229:1451-54.

21. Gunnar RM, Passamani ER, Bourdillon PD, et al. Guideline for the early management of patients with acute myocardial infarction. A report of the American College of Cardiology/ American Heart Association Task Force on assessment of diagnostic and therapeutic cardiovascular procedures. (Subcommittee to develop guidelines for the early management of patients with acute myocardial infarction. J Am Coll Cardiol 1990;16:249-92.

22. Hlarky MA, Boineau RE, Higginhorhan MB, et al. A brief self-administered questionnaire to determine functional capacity (the Duke Activity Status Index). Am J Cardiol 1989;64:651-54.

23. Goldman L, Hashimoto B, Cook EF, et al. Comparative reproductibility and validity of systems for assessing cardiovascular functional class: advantages of a new specific activity scale. Circulation 1981;64:1227-34.

24. Fletcher GF, Balady G, Froelicher VF, et al. Exercise standard. A statement for healthcare professionals from the American Heart Association. Circulation1995;91:580-615.

25. Isaacson $\mathbf{J} \mathbf{H}$. Preoperative atenolol reduced postoperative cardiac mortality. Cleveland Clin J Med 2001; 68: 273-74.

26. Auerbach AA, Goldman L. Beta blockers and reduction of cardiac events in noncardiac surgery. JAMA 2002; 287:143544

27. Robert D. Stevens Haran Burri Martin R. Tramèr Pharmacologic Myocardial Protection in Patients Undergoing Noncardiac Surgery: A Quantitative Systematic Review Anesth Analg 2003;97:623-33.

28. Roy A, Roy V. Using b-blockers to cut perioperative risk in CAD: cardioprotective strategies for noncardiac surgery. Postgrad Med 2005;118:34-42.

29. Opie LH, Gersch BJ. Drugs for the heart.6th ed. Pennsylvania:Elsevier Saunders, 2005.

30. Devereaux PJ, Beattie WS, Choi PT-L, et al. How strong is the evidence for the use of perioperative beta blockers in noncardiac surgery? Systematic review and meta-analysis of randomised controlled trials. Br Med J 2005; 331: 313-21.

31. Torsher LC, Shub C, Rettke SR, et al. Risk of patients with severe aortic stenosis undergoing to noncardiac surgery. Am J Cadiol 1998;81:448-52.

32. Reyes VP, Raju BS, Wynne J, et al. Percutaneous balloon valvuloplasty compared with open surgical commissurotomy for mitral stenosis. N Engl J Med 1994;331:961-67.

33. Mosley JC, Clarke JM, Ell PJ, et al. Assessment of myocardial function before aortic surgery by radionuclide angiocardiography. Br J Surg 1985;72:886-87.

34. Halm EA, Browner WS, Tubau JF, et al. Ecocardiography for assessing cardiac risk in patients having noncardiac surgery. Study of Peroperative Ischemia Research Group. Ann Inter Med 1996;125:435-41.

35. Mangano DT. Perioperative cardiac morbidity. Anesthesiology 1990;72:153-84.

36. Devereaux PJ. Perioperative cardiac risk assessment and modification in patients undergoing noncardiac surgery [workshop presentation]. Annual Conference of Canadian Society of Internal Medicine. October 2002; Halifax.

37. Lee TH, Marcantonio ER, Mangione CM, et al. Derivation and prospective validation of a single index for cardiac risk of major noncardiac surgery. Circulation 1999;100:1043-49.

38. Palda VA, Detsky AS. Perioperative assessment and management of risk from coronary artery disease. Ann Intern Med 1997;127:313-28.

39. Cruickshank JM. Beta-blocker continues surprise us. Eur Heart J 2000; 21:354-64

40. Devereaux PJ, Yusuf S, Yang H, et al. Are the recommendations to use perioperative â-blocker therapy in patients undergoing noncardiac surgery based on reliable evidence? CMAJ 2004;171:245-47.

41. Lindenauer P K, P Penelope, W Kaijun, et al. Perioperative beta-blocker therapy and mortality after major noncardiac surgery. N Engl J Med. 2005;353:349-61.

42. Poldermans D, and Boersma E. Beta-Blocker Therapy in Noncardiac Surgery. N Engl J Med. 2005;353:412-414.

43. Mangano DT, Layug EL, Wallace A, Tateo I. Effect of atenolol on mortality and cardiovascular morbidity after noncardiac surgery. N Engl J Med 1996;335:1713-20. [Erratum, N Engl J Med 1997;336:1039.]

44. Poldermans D, Boersma E, Bax JJ, et al. The effect of bisoprolol on perioperative mortality and myocardial infarction in high-risk patients undergoing vascular surgery. N Engl J Med 1999;341:1789-94.

45. Juul AB. Randomized, blinded trial on perioperative metoprolol versus placebo for diabetic patients undergoing noncardiac surgery. In: Late-breaking clinical trials I of the American Heart Association Scientific Sessions 2004, New Orleans, November 7-10, 2004. Abstract.

46. Eagle KA, Berger PB, Calkins H, et al. ACC/AHA guideline update for perioperative cardiovascular evaluation for non- 
cardiac surgery - executive summary. A report of the American College of Cardiology / American Heart Association Task Force on Practice Guidelines (Committee to Update the 1996 Guidelines on Perioperative Cardiovascular Evaluation of Noncardiac Surgery). J Am Coll Cardiol 2002; 39:542-53.

47. Bodenheimer T. An easy alliance: clinical investigators and the pharmaceutical industry. N Engl J Med 2000;342:153944.

48. McCrary SV, Anderson CB, Jakovljevic J, et al. A National survey of policies on disclosure of conflicts of interest in biomedical research. N Engl J Med 2000; 343:1621-26.
49. Stossel T. P. Regulating Academic -Industrial research relationships - Solving problems or stifling progress. N Engl J Med 2005; 353:1060-65.

\section{Endereço para correspondência:} JÚLIO CÉSAR M SANTOS JR Avenida Min. Urbano Marcondes, 110 12.515-230 - GUARATINGUETÁ (SP)

E-mail: instmed@provale.com.br 